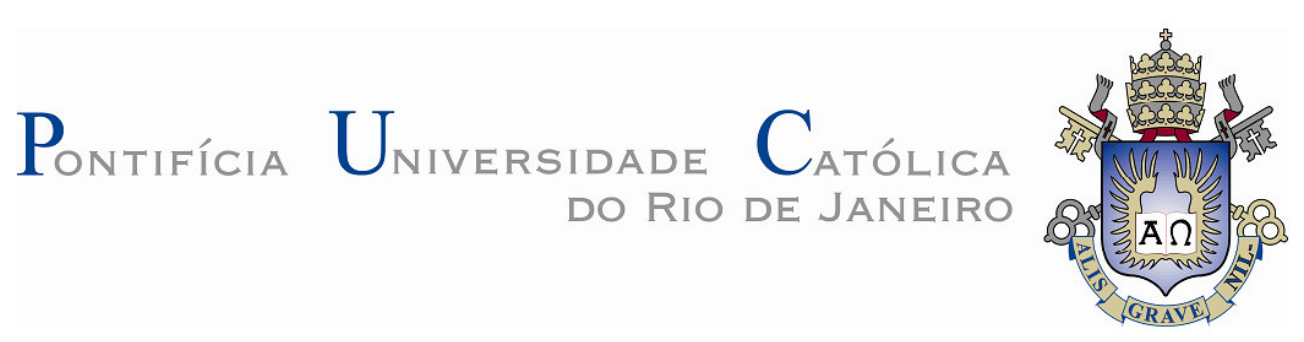

Cristiane Arantes Ferreira

\title{
Estudo do Comportamento Mecânico de Solos Reforçados com Fibras através do Método de Elementos Discretos
}

\section{Dissertação de Mestrado}

Dissertação apresentada como requisito parcial para obtenção do titulo de Mestre pelo Programa de Pós-Graduação em Engenharia Civil do Departamento de Engenharia Civil da PUCRio.

Orientador: Eurípedes do Amaral Vargas Junior Co-orientadora: Michéle Dal Toé Casagrande

Rio de Janeiro Junho de 2010 


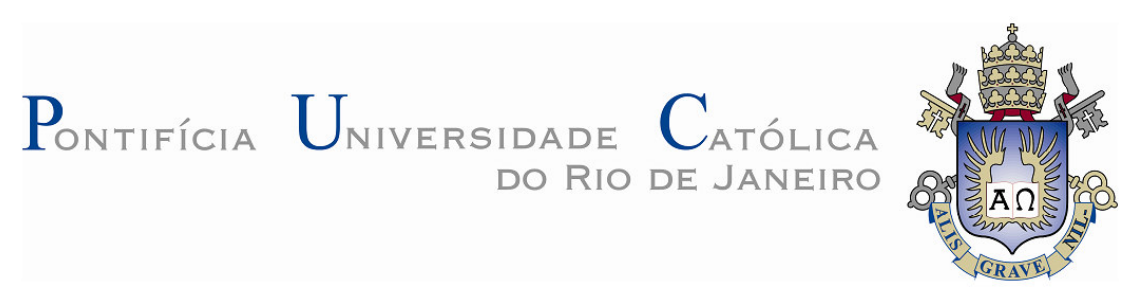

Cristiane Arantes Ferreira

\title{
Estudo do Comportamento Mecânico de Solos Reforçados com Fibras através do Método de Elementos Discretos
}

\begin{abstract}
Dissertação apresentada como requisito parcial para obtenção do titulo de Mestre pelo Programa de Pós-Graduação em Engenharia Civil do Departamento de Engenharia Civil da PUC.Rio. Aprovada pela Comissão Examinadora abaixo assinada.
\end{abstract}

Eurípedes do Amaral Vargas Júnior Orientador Departamento de Engenharia Civil - PUC-Rio

Michéle Dal Toé Casagrande Co-orientadora Departamento de Engenharia Civil - PUC-Rio

Nilo Cesar Consoli UFRGS

Tácio Mauro Pereira de Campos Departamento de Engenharia Civil - PUC-Rio

José Eugênio Leal Coordenador Setorial do Centro

Técnico Científico - PUC-Rio

Rio de Janeiro, 06 de junho de 2010 
Todos os direitos reservados. É proibida a reprodução total ou parcial do trabalho sem autorização da universidade, do autor e da orientadora.

\section{Cristiane Arantes Ferreira}

Graduou-se em Engenharia Civil pela Universidade Federal de Juiz de Fora (UFJF) em 2007. Ingressou no mestrado na Pontifícia Universidade Católica do Rio de Janeiro em 2007, desenvolvendo Dissertação na linha de pesquisa de Geotecnia aplicada a reforço de solos.

Ficha Catalográfica

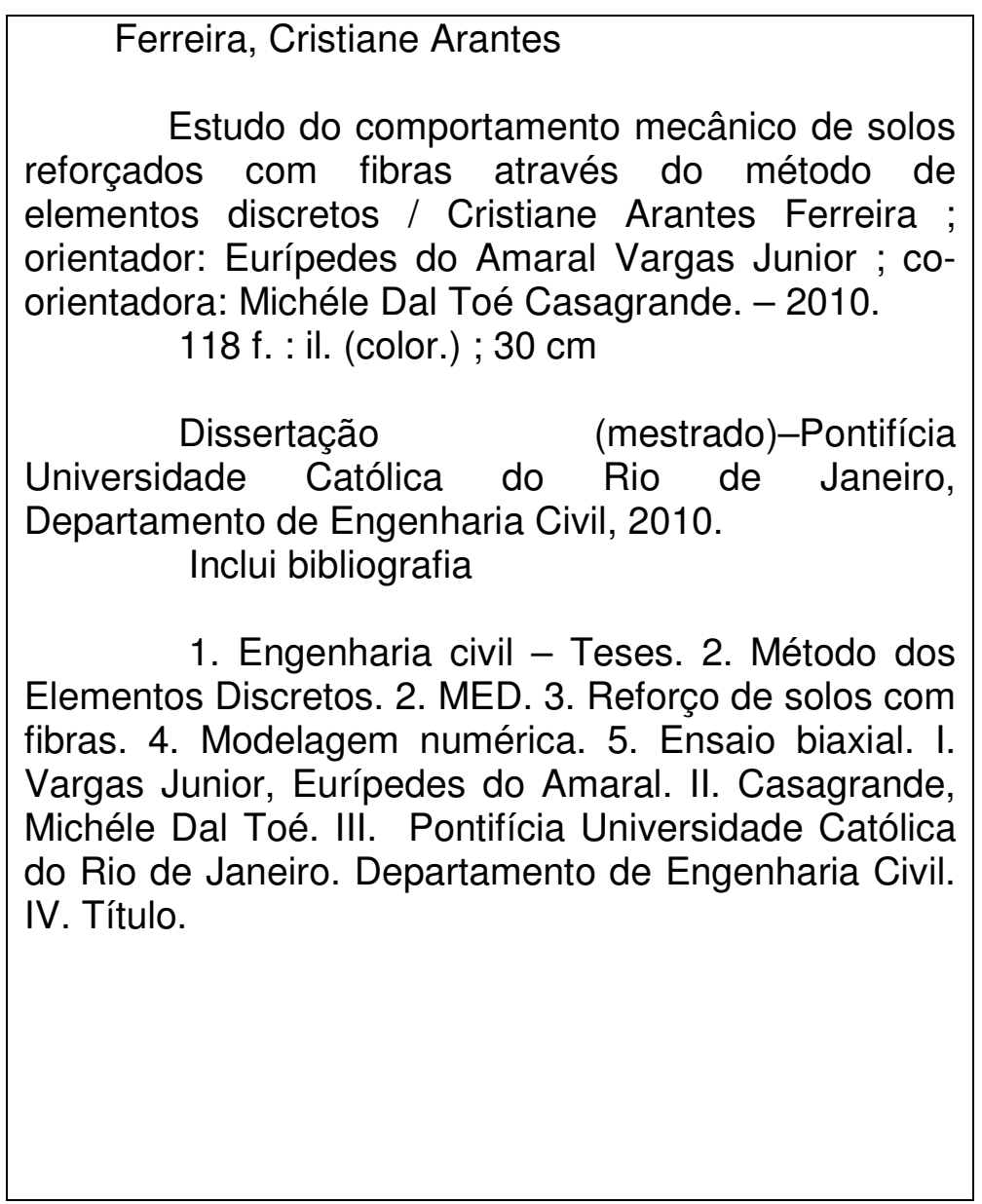

CDD: 624 
Dedico esta Dissertação à minha família, em especial aos meus pais,

Paulo e Vanda. 


\section{Agradecimento}

À Deus, criador de todas as coisas, por iluminar meu caminho e pela força para superar grandes obstáculos. Sem ele nada disso seria possível.

Aos meus pais, Vanda e Paulo por me proporcionar tudo o que tenho e o que sou pelo amor incondicional e total apoio durante toda a minha vida. Aos meus irmãos Vinícius e Bianca, pela amizade e carinho. A toda família, pela bondade e incentivo. Ao Fabinho, pelo companheirismo.

À minha amiga Fernanda por entender minha ausência.

A duas pessoas muito especiais presentes em todo o processo desta dissertação: Raquel Velloso e Michéle Casagrande. Não tenho palavras para expressar meu agradecimento.

Aos todos os meus amigos da "favelinha" na Puc-Rio, em especial ao Danilo, ao David, a Carla, ao Luis e a Luciana.

A todos os meus amigos da UFJF (Universidade Federal de Juiz de Fora), e aos colegas de trabalho que de uma forma ou de outra me estimularam ou me ajudaram.

Ao Departamento de Engenharia Civil da PUC-Rio, em especial a Rita, pelo excelente atendimento aos professores e aos alunos.

À minha amiga Michela que além de dividir as contas comigo sempre me proporciona bons e descontraídos momentos além de excelentes conselhos.

À minha prima Amanda por me acolher quando cheguei ao Rio de Janeiro.

Ao Professor Eurípedes Vargas, orientador deste trabalho, a quem admiro por ser excelente professor e pesquisador.

A CAPES pelo apoio financeiro.

E finalmente a Sandra, excelente profissional, muito importante nesta etapa final. 


\section{Resumo}

Ferreira, Cristiane Arantes; Vargas Júnior, Eurípedes do Amaral (Orientador); Casagrande, Michéle Dal Toé (Co-Orientadora). Estudo do Comportamento Mecânico de Solos Reforçados com Fibras através do Método de Elementos Discretos. Rio de Janeiro, 2010. 118p. Dissertação de Mestrado - Departamento de Engenharia Civil, Pontifícia Universidade Católica do Rio de Janeiro.

Um grande número de novos materiais geotécnicos foi desenvolvido baseado na adição de materiais fibrosos, sendo incorporados como elementos de reforço. A técnica de solo reforçado pode ser representada pela produção e aplicação, não somente de fibra natural, mas também de fibras sintéticas e poliméricas. Estudos anteriores de solos reforçados com fibras de polipropileno têm mostrado melhora significativa das propriedades mecânicas dos solos, tais como o aumento da resistência de pico e resistência pós-pico, ductilidade e tenacidade. Estes resultados mostram um grande potencial deste tipo de fibra, quando utilizado como reforço de solos, por exemplo, em base de fundações superficiais, aterros sobre solos moles e liners de cobertura de aterros sanitários. A partir de ajustes matemáticos para determinar a interação entre solos granulares e observações do comportamento global em macro-escala tornou possível analisar o comportamento de solos granulares reforçados com fibras de uma forma micro-mecânica. A modelagem numérica do comportamento mecânico de solos reforçados com fibras de polipropileno, através de uma análise micro-mecânica, utiliza como ferramenta o Método dos Elementos Discretos (MED), que permite a representação do solo em $2 \mathrm{D}$, a partir de um conjunto de partículas de elementos discretos circulares. O MED descarta a visão clássica do solo como uma forma contínua, proporcionando a possibilidade de modelá-lo como partículas constituintes. Sua formulação baseia-se no equilíbrio de forças e de deslocamentos gerados pelos contatos, os quais são descritos através das leis da física clássica, permitindo o mapeamento dos movimentos de cada partícula. A vantagem da micro-mecânica é a possibilidade de explicitar microestruturas, tais como fibras de polipropileno, responsáveis pela mudança no comportamento do solo. Com base no estudo deste fenômeno, 
causado pela inserção de fibras de polipropileno em materiais granulares, formulações matemáticas foram propostas com a finalidade de descrever o comportamento de solos reforçados através da implementação do código de elementos discretos (DEMlib). Após a calibração e validação do programa, a influência decorrente da inserção do reforço de fibra ao solo foi analisada, sendo realizadas simulações de ensaios biaxiais em amostras discretas de areia, com e sem o reforço fibroso. O comportamento micro-mecânico de misturas reforçadas permitiu avaliar os efeitos das mudanças no teor de fibras presente na matriz de solo, bem como diferentes rigidezes das fibras. Conclui-se que o estudo realizado pelo Método dos Elementos Discretos identificou a real interação entre as partículas do solo e do reforço em forma de fibra, indicando que as fibras, quando inseridas no solo, podem sofrer deformações plásticas de tração e alongamento até atingir a ruptura, proporcionando a melhora nos parâmetros mecânicos do solo.

\section{Palavras-chave}

Método dos Elementos Discretos; MED; reforço de solos com fibras; modelagem numérica; ensaio biaxial. 


\section{ABSTRACT}

Ferreira, Cristiane Arantes; Vargas Júnior, Eurípedes do Amaral (Advisor); Casagrande, Michéle Dal Toé (Co-Advisor). Study of Mechanical Behavior of Fiber Reinforced Soil through Discrete Element Method. Rio de Janeiro, 2010. 118p. MSc Dissertation - Civil Engineering Department, Pontifical Catholic University of Rio de Janeiro.

A large number of new geotechnical materials was developed based on the addition of fibrous materials being incorporated as reinforcement. The technique of reinforced soil can be represented by the production and application, not only natural fiber, but also synthetic fibers and polymer. Previous studies of soil reinforced with polypropylene fibers have shown significant improvement of mechanical properties of soils, such as increasing the resistance peak and postpeak strength, ductility and toughness. These results show a great potential for this type of fiber, when used as soil reinforcement, for example, based on shallow foundations, embankments over soft soils and liners for landfill cover. From mathematical adjustments to determine the interaction between granular soils and the observation of global macro-scale become possible to analyze the behavior of granular soils reinforced with fibers in a micro-mechanics. The numerical modeling of mechanical behavior of soil reinforced with polypropylene fibers, through a micro-mechanical analysis, the tool uses as the Discrete Element Method (DEM), which allows the representation of the soil in $2 \mathrm{D}$, from a set of particles circular discrete elements. The MED rule out the classical view of soil as a continuous form, providing the ability to model it as a constituent particle. Its formulation is based on the balance of forces and displacements generated by the contacts, which are explained through the laws of classical physics, allowing the mapping of movements of each particle. The advantage of micro-mechanics is the possibility of explicit microstructures, such as polypropylene fibers, responsible for the change in the behavior of the soil. Based on the study of this phenomenon, caused by the insertion of polypropylene fibers in granular materials, mathematical formulations have been proposed in order to describe the behavior of reinforced soils through the implementation of the Code of discrete elements (DEMlib). After calibration and 
validation program, the influence due to the insertion of fiber reinforcement to the soil was analyzed, and simulations of biaxial tests on discrete samples of sand, with and without the fibrous reinforcement. The micro-mechanical behavior of blends reinforced allowed evaluating the effects of changes in fiber content present in the soil matrix and different rigidities of the fibers. We conclude that the study by the Discrete Element Method identified the actual interaction between the soil particles and the reinforcement in the form of fiber, indicating that the fibers, when inserted into the soil, may undergo plastic deformation and tensile elongation until the rupture, providing an improvement in mechanical parameters of soil.

\section{Keywords}

Discrete Element Method; DEM; soil reinforcement with fibers; numerical modeling; biaxial test. 


\section{Sumário}

Lista de Figuras

Lista de Tabelas

Lista de Símbolos

1. INTRODUÇÃO 19

1.1. INTRODUÇÃO E DEFINIÇÃO DO PROBLEMA 19

1.2. OBJETIVOS 21

1.3. ORGANIZAÇÃO DA PESQUISA 22

2. REFORÇO DE SOLOS COM FIBRAS 23

2.1 CONSIDERAÇÕES INICIAIS 23

2.2 SOLOS REFORÇADOS 23

2.3 REFORÇO COM FIBRAS 26

2.3.1 Fibras Naturais 26

2.3.2 Fibras Minerais 27

2.3.3 Fibras Metálicas $\quad 27$

2.3.4 Fibras Poliméricas 27

2.4 INTERAÇÃO SOLO-FIBRA 29

2.5 COMPORTAMENTO DOS SOLOS REFORÇADOS COM FIBRAS 32

2.6 TEORIA DO ESTADO CRÍTICO 34

2.6.1 Conceitos Fundamentais 34

3. MODELAGEM MICRO - MECÂNICA DISCRETA DE SOLOS GRANULARES 43

3.1 CONSIDERAÇÕES INICIAIS 43

3.2 MODELAGEM MICRO-MECÂNICA 43

3.3 DIFERENÇA ENTRE MODELAGEM CONTÍNUA E DISCRETA 44 
3.4 MÉTODO DOS ELEMENTOS DISCRETOS

3.4.1 Cinemática do Elemento 47

3.4.2 Leis da Física Clássica 47

3.4.3 Amortecimento e Dissipação de Energia Erro! Indicador não definido.

3.4.4 Integração Numérica

4. IMPLEMENTAÇÃO COMPUTACIONAL 58

4.1. GERAÇÃO DO MODELO 58

4.2. PESQUISA POR PARES DE CONTATO 60

4.3. CICLO COMPUTACIONAL 62

5. MODELAGEM MICRO-MECÂNICA DISCRETA DE SOLOS GRANULARES REFORÇADOS COM FIBRAS 63

5.1. CONSIDERAÇÕES INICIAIS 63

5.2. SIMULAÇÃO DE ENSAIOS LABORATORIAIS 63

5.3. ENSAIO BIAXIAL 64

5.3.1 Considerações sobre o modelo numérico 64

5.3.2 Calibração dos parâmetros 65

5.3.3 Calibração do Ensaio Biaxial 73

5.3.4 Representação das Fibras 83

5.4. ENSAIO COMPRESSÂO HIDROSTÁTICA 101

6. CONSIDERAÇÕES FINAIS 106

6.1 CONCLUSÕES 106

6.2 SUGESTÕES PARA TRABALHOS FUTUROS 108

REFERÊNCIAS BIBLIOGRÁFICAS 109 


\section{Lista de Figuras}

Figura 1:Comparação de Estaca com elemento de reforço de solo

(McGown et al., 1978)

Figura 2: Linha Isotrópica de Compressão e Linha do Estada Crítico (

Atkinson, 1993)

Figura 3: (a) Representação da envoltória de pico e (b) da Superfície

Limite de Estado. (Atkinson, 1993)

Figura 4: (a)Parâmetros para normalização (Atkinson, 1993) e (b)

Superfície Limite de Estado no plano (Atkinson \& Bransby, 1978)

Figura 5: (a)Representação superfície limite de estado no plano e (b) no espaço (Atkinson \& Bransby, 1978)

Figura 6: Relação entre q/p' e a taxa de dilatação (Atkinson, 1993) 42

Figura 7: Cinemática de um elemento esférico

Figura 8: Sistema de referênca local para o contato entre dois emelentos esféricos

Figura 9: Sistema de coordenadas Local e Global (Huaman, 2008) 49

Figura 10: Penetração no contato entre elementos 50

Figura 11: Penetração no contato entre elementos e parede $\quad 50$

Figura 12: Modelo da força de contato

Figura 13: Esquema do mecanismo de força-deslocamento entre dois discos (ITASCA, Online Manual Table of Contents, Version 3.0, 1996) 52 Figura 14: Esquema de mecanismo de força-deslocamento entre parede e disco (ITASCA, Online Manual Table of Contents, Version 3.0, 1996) 52 Figura 15: Plano Tensão x Deformação de um compósitos reforçado com fibras, (Michalowski and Zhao - Falure of fiber-reinforced Granular Soils 1996)

Figura 16: Modelo em 2D de uma amostra de solo reforçado

Figura 17: Modelo em 2D de uma amostra de solo reforçado

Figura 18: Ciclo computacional 
Figura 19: Esquema do Modelo de Contato entre dois discos

Figura 20: Simulação de Ensaio Biaxial com diferentes modelos de contatopara tensão confinante de 100kPa: (a) Curva Tensão x Deformação axial; (b) Curva Deformação volumétrica x Deformação axial.

Figura 21: Simulação de Ensaio Biaxial com diferentes modelos de contato para tensão confinante de 200kPa: (a) Curva Tensão x Deformação axial; (b) Curva Deformação volumétrica x Deformação axial.

Figura 22: Simulação de Ensaio Biaxial com diferentes modelos de contato para tensão confinate de 400kPa: (a) Curva Tensão x Deformação axial; (b) Curva Deformação volumétrica x Deformação axial

Figura 23: Trajetória de tensão das simulações de areias fofa e densa 72 Figura 24: Simulação de Ensaio Biaxial com areia fofa: (a) Curva Tensão $x$ Deformação axial; (b) Curva Deformação volumétrica x Deformação axial

Figura 25: Simulação de Ensaio Biaxial com com areia compacta: (a) Curva Tensão x Deformação axial; (b) Curva Deformação volumétrica $x$ Deformação axial

Figura 26: Trajetória de tensão das simulações de areias fofa e densa 76 Figura 27: Curvas Granulométricas

Figura 28: Ensaio biaxial em amostras de solo graduado e uniforme para tensão confinante de 100kPa: (a) Curva tensão desviadora $x$ deslocamento vertical; (b) Curva deslocamento volumétrico $\mathrm{x}$ deslocamento vertical

Figura 29: Ensaio biaxial em amostras de solo graduado e uniforme para tensão confinante de 200kPa: (a) Curva tensão desviadora $x$ deslocamento vertical; (b) Curva deslocamento volumétrico $\mathrm{x}$ deslocamento vertical

Figura 30: Ensaio biaxial em amostra graduada: a) Arranjo inicial; b)

Detalhe do arranjo denso e estável 83

Figura 31: Disposição fibra-fissura idealizada (Taylor, 1994)

Figura 32: Interação fibra-elemento circular 
Figura 33: Curva Tensão x Deformação axial - Variação da Rigidez do elemento de reforço

Figura 34: Curvas tensão e deformação volumétrica x distorção em ensaios traxiais drenados - compressão axial, para (a) areia e (b) areia-fibra. (Casagrande, 2005)

Figura 35: Curvas tensão e deformação volumétrica $x$ distorção em ensaios traxiais drenados - compressão axial, para (a) arenito e (b) arenito-fibra. (Casagrande, 2001 e Heineck, 2002)

Figura 36: Ensaio biaxial em amostras de solo granular - areia: (a) Curva tensão desviadora $\mathrm{x}$ deslocamento vertical; (b) Curva deslocamento volumétrico $\mathrm{x}$ deslocamento vertical

Figura 37: Ensaio biaxial em amostras de solo granular - areia + fibra: (a) Curva tensão desviadora $x$ deslocamento vertical; (b) Curva deslocamento volumétrico $\mathrm{x}$ deslocamento vertical

Figura 38: Envoltórias e parâmetros de resistência ao cisalhamento $\left(\varepsilon_{\mathrm{a}}=\right.$ 10\%) (a) areia e (b) areia-fibra 96

Figura 39: Comparação entre as envoltórias de resistência obtidas $\quad 97$ Figura 40: Superfície de estado limite para (a) areia e (b) areia-fibra $\quad 99$ Figura 41: Superfície de estado limite para areia e areia-fibra 100 Figura 42: Ensaio de compressão hidrostática:(a) Curva tensão de confinamento $x$ deformação volumétrica da Areia; (b) Curva tensão de confinamento $x$ deformação volumétrica da Areia + fibra

Figura 43: Ensaio de compressão hidrostática em matriz granular com fibra: a) Arranjo inicial (Matriz granular + Fibra); b) Detalhe dos Liks: com coesão e sem coesão

Figura 44: Ensaio de compressão hidrostática: Curva tensão de confinamento $\mathrm{x}$ deformação volumétrica 


\section{Lista de Tabelas}

Tabela 1: Modelos de Contato 68

Tabela 2: Índices físicos da areia de Osório 78

Tabela 3: Índices físicos - Elementos Discretos 79

Tabela 4: Característocas das Fibras - Elementos Discretos 86 


\section{Lista de Símbolos}

\begin{tabular}{|c|c|}
\hline ، & Relativo a tensões efetivas \\
\hline$\delta$ & Relativo a incrementos \\
\hline$\varepsilon_{\mathrm{a}}$ & Deformação axial \\
\hline$\varepsilon_{\mathrm{v}}$ & Deformação volumétrica \\
\hline$\varphi$ & Ângulo de atrito interno \\
\hline$\varphi_{\mathrm{c}}^{\prime}$ & $\begin{array}{l}\text { Ângulo de atrito interno no estado } \\
\text { crítico }\end{array}$ \\
\hline$\gamma$ & Deformação cisalhante \\
\hline$\gamma_{\mathrm{d}}$ & Peso específico aparente seco \\
\hline$v$ & Coeficiente de Poisson \\
\hline$\lambda$ & $\begin{array}{l}\text { Declividade da linha isotrópica de } \\
\text { compressão no plano v:ln p' }\end{array}$ \\
\hline$\Gamma$ & $\begin{array}{l}\text { Volume específico ou intercepto da } \\
\text { LEC para p'=1kPa, no plano v:ln p' }\end{array}$ \\
\hline$\tau$ & Tensão de cisalhamento \\
\hline$\rho$ & Massa específica do solo \\
\hline$\sigma_{\mathrm{v}}$ & Tensão vertical \\
\hline$\sigma_{1,} \sigma_{3}$ & Tensões principais maior e menor \\
\hline$v$ & Volume específico \\
\hline$\eta$ & $\begin{array}{l}\text { Inclinação da LIC no plano } \\
\text { normalizado } q / p^{\prime}: p^{\prime} / p 1_{e}\end{array}$ \\
\hline$\psi$ & $\begin{array}{l}\text { Parâmetro de estado (Been \& } \\
\text { Jefferies, 1985) }\end{array}$ \\
\hline$\kappa$ & $\begin{array}{l}\text { Declividade da linha de expansão no } \\
\text { plano } v \text { :ln p' }\end{array}$ \\
\hline$\omega$ & Teor de umidade \\
\hline$\omega_{\text {ótimo }}$ & $\begin{array}{l}\text { Teor de umidade ótimo de } \\
\text { compactação }\end{array}$ \\
\hline$\delta \mathrm{h}$ & Deslocamento horizontal \\
\hline $\mathrm{ABNT}$ & $\begin{array}{l}\text { Associação Brasileira de Normas } \\
\text { Técnicas }\end{array}$ \\
\hline ASTM & $\begin{array}{l}\text { American Society for Testing and } \\
\text { Materials }\end{array}$ \\
\hline $\mathrm{B}$ & $\begin{array}{l}\text { Parâmetro de poro-pressão de } \\
\text { Skempton }\end{array}$ \\
\hline $\mathrm{c}$ & Coesão \\
\hline CBR & California Bearing Ratio \\
\hline $\mathrm{Cu}$ & Coeficiente de uniformidade \\
\hline $\mathrm{Cv}$ & Coeficiente de adensamento \\
\hline $\mathrm{d}$ & Diâmetro da fibra \\
\hline
\end{tabular}




\begin{tabular}{|c|c|}
\hline D10 & Diâmetro efetivo das partículas \\
\hline D50 & Diâmetro médio \\
\hline dtex & $\begin{array}{l}\text { Unidade que representa a espessura } \\
\text { dos filamentos }(1 \mathrm{dtex}=1 \mathrm{~g} / 10000 \mathrm{~m}) \text {. }\end{array}$ \\
\hline $\mathrm{e}$ & Índice de vazios \\
\hline $\mathrm{E}$ & $\begin{array}{l}\text { Módulo de deformabilidade ou } \\
\text { Módulo de Young }\end{array}$ \\
\hline $\mathrm{e}_{0}$ & Índice de vazios inicial \\
\hline $\mathrm{F}_{\mathrm{t}}$ & Resistência à tração da fibra \\
\hline G & Módulo de cisalhamento \\
\hline $\mathrm{G}_{0}$ & $\begin{array}{l}\text { Módulo de cisalhamento inicial ou } \\
\text { elástico }\end{array}$ \\
\hline $\mathrm{G}_{\text {máx }}$ & Módulo de cisalhamento máximo \\
\hline LEC & Linha do Estado Crítico \\
\hline LIC & Linha Isotrópica de Compressão \\
\hline LL & Limite de liquidez \\
\hline MED & Método dos Elementos Discretos \\
\hline M & Massa \\
\hline $\mathrm{C}$ & Amortecimento \\
\hline $\mathrm{S}$ & Rigidez \\
\hline$\Delta \mathrm{X}$ & Vetor Deslocamento \\
\hline$\Delta \mathrm{F}$ & Vetor Força \\
\hline ri & Vetor posição \\
\hline vi & Velocidade linea \\
\hline wi & Velocidade angular \\
\hline $\mathrm{t}$ & tempo \\
\hline$\Delta \mathrm{t}$. & Intervalo de tempo \\
\hline $\operatorname{dij}$ & Impenetrabilidade entre partículas \\
\hline dpkj & $\begin{array}{l}\text { Impenetrabilidade entre partícula e } \\
\text { parede }\end{array}$ \\
\hline Un & Deslocamento relativo \\
\hline$K^{n}$ & Rigidez normal \\
\hline$K^{t}$ & Rigidez tangencial \\
\hline $\mathrm{Cn}$ & Amortecimento noral \\
\hline $\mathrm{Ct}$ & Amortecimento tangencial \\
\hline$n_{i}$ & Normal ao plano de contato \\
\hline$x_{i}^{[A]}$ & $\begin{array}{l}\text { Vetores posição dos centros dos } \\
\text { discos }\end{array}$ \\
\hline$x_{i}^{[E]}$ & $\begin{array}{l}\text { Vetores posição dos centros dos } \\
\text { discos }\end{array}$ \\
\hline$d$ & Distancia entre os centros \\
\hline$V^{S}$ & Velocidade cisalhante \\
\hline$x_{i}^{[+j]}$ & Velocidade translacional \\
\hline$\omega_{3}^{[\Phi]}$ & Velocidade rotacional, \\
\hline
\end{tabular}




$\begin{array}{cl}\mu & \text { Coeficiente de atrito } \\ \mathrm{F}_{i} & \text { Força de translação } \\ g_{i} & \text { Gravidade } \\ m & \\ \mathrm{M}_{i} & \text { Massa } \\ \dot{H}_{i} & \text { Resultante do momento atuante } \\ I_{i} & \text { Momento angular da partícula } \\ \dot{\omega}_{1} & \text { Momento de Inércia } \\ F_{d a m} & \text { Acelerações angulares } \\ T+\Delta t, & \text { Força de amortecimento } \\ M_{m i} & \text { Incrementos de tempo } \\ K_{m a ́ x} & \text { Menor massa } \\ & \text { Maior constante elástica normal ou } \\ & \text { tangencial }\end{array}$

